\title{
Volume Potential-Integral-Equation Formulation for Electromagnetic Scattering by Dielectric Objects
}

\section{Markkanen, Johannes}

IEEE

2016

Markkanen , J 2016 , Volume Potential-Integral-Equation Formulation for Electromagnetic

Scattering by Dielectric Objects . in 2016 URSI INTERNATIONAL SYMPOSIUM ON

ELECTROMAGNETIC THEORY (EMTS) . URSI International Symposium on

Electromagnetic Theory , IEEE , New York , pp. 468-471, URSI International Symposium on

Electromagnetic Theory , Espoo , Finland , 14/08/2016 . https://doi.org/10.1109/URSI-EMTS.2016.7571428

http://hdl.handle.net/10138/228804

https://doi.org/10.1109/URSI-EMTS.2016.7571428

submittedVersion

Downloaded from Helda, University of Helsinki institutional repository.

This is an electronic reprint of the original article.

This reprint may differ from the original in pagination and typographic detail.

Please cite the original version. 


\title{
Volume Potential-Integral-Equation Formulation for Electromagnetic Scattering by Dielectric Objects
}

\author{
Johannes Markkanen* \\ *Department of Physics, University of Helsinki, Finland \\ e-mail: johannes.markkanen@helsinki.fi
}

\begin{abstract}
A volume potential-integral-equation formulation for electromagnetic scattering by dielectric objects is developed and discretized with fully continuous nodal basis functions. The equations are tested with either the point-matching or Galerkin's testing procedure. Galerkin's testing shows superior accuracy over the point-matching as well as over the standard discretization of the electric field volume integral equation with SchaubertWilton-Glisson (SWG) functions. The potential formulation is accelerated by the precorrected-FFT method.
\end{abstract}

\section{INTRODUCTION}

Electromagnetic scattering by strongly inhomogenous objects is a challenging problem arising from many different fields in science and engineering such as remote sensing, electromagnetic imaging, bioelectromagnetics, etc. The volumeintegral-equation (VIE) methods are typically applied for such problems in addition to the finite-element (FEM) and the finitedifference time-domain (FDTD) methods.

Many different VIE formulations exist. The most common ones are written for fields [1], flux densities [2], and polarization currents [3], [4]. The potential volume-integral-equation formulation (PVIE) has been derived in [5]. In the PVIE, the unknown functions are fully continuous scalar and vector potentials whereas in other formulations the unknowns are partly or fully discontinuous. This has a significance impact on numerical properties of the discretized system.

In [5], [6], the PVIE was discretized with curvilinear cubes in combination with nodal Lagrange basis functions. Further, the point-matching method was applied to convert the integral equations into a system of linear equations. The accuracy was found to be good, however, the PVIE has not gain much attention since it was introduced. In [7], the equation for the scalar potential was replaced by the discretized Lorenz gauge condition. This approach, however, has shown some serious accuracy problems [8]. Recently, the spectral properties of various VIE formulations were studies in [9] and the potential formulation showed some promising characteristics from the preconditioning point of view. The discrete spectrum of the potential formulation coincides almost exactly with the theoretically derived spectrum whereas the discrete spectra of other formulations deviate from the predicted ones. Unfortunately, the accuracy of the PVIE was shown to be much worse than any other formulations when it was discretized with tetrahedral elements and the point-matching scheme.
In this paper, we show that the accuracy of the PVIE can be improved by writing the equations for the vector potential and its divergence in combination with the Lorenz gauge condition. Further, it is shown that Galerkin's testing procedure leads to superior accuracy compared to the poing-matching approach.

\section{Potential formulation}

Consider time-harmonic $\left(e^{-i \omega t}\right)$ electromagnetic scattering by a three-dimensional object $D$ in a homogeneous background medium with constants $\epsilon_{0}$ and $\mu_{0}$. The object $D$ is assumed to be isotropic and characterized by the position dependent relative permittivity function $\epsilon_{r}(\boldsymbol{r})$. Electromagnetic fields can be represented in terms of the vector $\boldsymbol{A}$ and scalar $V$ potentials as

$$
\boldsymbol{E}=i \omega \boldsymbol{A}-\nabla V, \quad \boldsymbol{H}=\frac{1}{\mu_{0}} \nabla \times \boldsymbol{A} .
$$

To define the potentials ambiguously, a gauge condition must be imposed for the divergence of the vector potential. Here, we use the Lorenz gauge

$$
i \omega \nabla \cdot \boldsymbol{A}=-k^{2} V
$$

With the Lorenz gauge imposed, both potentials satisfy the Helmholtz equation:

$$
\begin{aligned}
\nabla^{2} \boldsymbol{A}+k^{2} \boldsymbol{A} & =-\mu_{0} \boldsymbol{J}, \\
\nabla^{2} V+k^{2} V & =-\frac{\rho}{\epsilon_{0}},
\end{aligned}
$$

whose solutions in terms of the free-space Green's functions $G$ read as

$$
\boldsymbol{A}=\mu_{0} \int_{D} G \boldsymbol{J} \mathrm{d} V
$$

and

$$
V=\frac{1}{\epsilon_{0}} \int_{D} G \rho \mathrm{d} V,
$$

where $\boldsymbol{J}$ and $\rho$ are the source current and charge densities, respectively.

To derive the potential formulation, we apply the volumeequivalence principle, i.e., the scatterer is removed and replaced by the equivalent current and charge densities

$$
\begin{gathered}
\boldsymbol{J}_{e q}=-i \omega \epsilon_{0}\left(\epsilon_{r}-1\right) \boldsymbol{E} \\
\rho_{e q}=\frac{1}{i \omega} \nabla \cdot \boldsymbol{J}_{e q} .
\end{gathered}
$$


The equivalent current and charge densities are the sources for the scattered potentials given by

$$
\begin{aligned}
\boldsymbol{A}^{s} & =\mu_{0} \int_{D} G \boldsymbol{J}_{e q} \mathrm{~d} V \\
V^{s} & =\frac{1}{\epsilon_{0}} \int_{D} G \rho_{e q} \mathrm{~d} V
\end{aligned}
$$

and the total potentials can be represented as a sum of incident and scattered potentials as

$$
\begin{aligned}
& \boldsymbol{A}=\boldsymbol{A}^{i n c}+\boldsymbol{A}^{s} . \\
& V=V^{i n c}+V^{s} .
\end{aligned}
$$

Substituting (9) into (11) and using the definition of the equivalent current, the integral equation for the vector potential reads as

$$
i \omega \boldsymbol{A}^{i n c}=i \omega \boldsymbol{A}-k^{2} \int_{D}\left(\epsilon_{r}-1\right)\left(i \omega \boldsymbol{A}-\nabla^{\prime} V\right) G \mathrm{~d} V .
$$

Taking the divergence of the above equation and using the Lorenz gauge (2), the equation for the scalar potential is obtained:

$$
V^{i n c}=V+\nabla \cdot \int_{D}\left(\epsilon_{r}-1\right)\left(i \omega \boldsymbol{A}-\nabla^{\prime} V\right) G \mathrm{~d} V .
$$

Next, by moving the derivative into the Green's function and since $\nabla G=-\nabla^{\prime} G$ we get

$$
V^{i n c}=V-\int_{D}\left(\epsilon_{r}-1\right)\left(i \omega \boldsymbol{A}-\nabla^{\prime} V\right) \cdot \nabla^{\prime} G \mathrm{~d} V
$$

and the potential formulation suitable for discretization can be written as

$$
\left\{\begin{aligned}
i \omega \boldsymbol{A}^{i n c} & =i \omega \boldsymbol{A}-k^{2} \int_{D}\left(\epsilon_{r}-1\right)\left(i \omega \boldsymbol{A}-\nabla^{\prime} V\right) G \mathrm{~d} V \\
V^{i n c} & =V-\int_{D}\left(\epsilon_{r}-1\right)\left(i \omega \boldsymbol{A}-\nabla^{\prime} V\right) \cdot \nabla^{\prime} G \mathrm{~d} V .
\end{aligned}\right.
$$

By defining the normalized vector potential $\tilde{\boldsymbol{A}}=i \omega \boldsymbol{A}$ improves numerical stability due to the better balance of the unknowns

$$
\left\{\begin{aligned}
\tilde{\boldsymbol{A}}^{i n c} & =\tilde{\boldsymbol{A}}-k^{2} \int_{D}\left(\epsilon_{r}-1\right)\left(\tilde{\boldsymbol{A}}-\nabla^{\prime} V\right) G \mathrm{~d} V \\
V^{i n c} & =V-\int_{D}\left(\epsilon_{r}-1\right)\left(\tilde{\boldsymbol{A}}-\nabla^{\prime} V\right) \cdot \nabla^{\prime} G \mathrm{~d} V .
\end{aligned}\right.
$$

Further, the equation for the scalar potential can be written as

$$
\begin{aligned}
V^{i n c} & =V+\int_{D} \nabla^{\prime} \cdot\left[\left(\epsilon_{r}-1\right)\left(\tilde{\boldsymbol{A}}-\nabla^{\prime} V\right)\right] G \mathrm{~d} V \\
& -\int_{\partial D} \boldsymbol{n}^{\prime} \cdot\left(\epsilon_{r}-1\right)\left(\tilde{\boldsymbol{A}}-\nabla^{\prime} V\right) G \mathrm{~d} S .
\end{aligned}
$$

The surface and volume integrals in (18) correspond contributions of the surface and volume charges, respectively. We note that the potential formulation derived here is exactly the same as that of in [5] (excluding normalization). In the discretized potential formulations in [5], [9], the volume integral was assumed to be zero inside each element and dropped out. This is a correct assumption for a constant permittivity. However, if the basis functions used for expanding the unknown potentials do not explicitly satisfy the zero divergence condition, there is no guarantee that the volume charge converges. This explains convergence problems of the PVIE observed in [9].

\section{DiscretizATION}

To discretize the potential integral equation, the domain is divided into tetrahedral elements. Since the integral equations for the vector and scalar potentials define bounded mappings:

$$
\begin{aligned}
& \boldsymbol{H}^{1}\left(\mathbb{R}^{3}\right)^{3} \times H^{1}\left(\mathbb{R}^{3}\right)^{1} \rightarrow \boldsymbol{H}^{1}\left(\mathbb{R}^{3}\right)^{3} \\
& \boldsymbol{H}^{1}\left(\mathbb{R}^{3}\right)^{3} \times H^{1}\left(\mathbb{R}^{3}\right)^{1} \rightarrow H^{1}\left(\mathbb{R}^{3}\right)^{1},
\end{aligned}
$$

the unknowns can be expanded with fully continuous basis functions except on the boundary $\partial D$. The scalar potential is expanded by linear nodal basis functions $N_{n}$ which span the finite-dimensional $H^{1}(D)$ space as

$$
V \approx \sum_{n} c_{n} N_{n}
$$

where $c_{n}$ are the unknown coefficients. On $\partial D$, "half" basis functions are used. Furthermore, each component of the vector potential $\boldsymbol{A}$ is expanded by the same functions as

$$
\begin{aligned}
& A_{x} \approx \sum_{n} x_{n} N_{n} \\
& A_{y} \approx \sum_{n} y_{n} N_{n} \\
& A_{z} \approx \sum_{n}^{n} z_{n} N_{n} .
\end{aligned}
$$

By applying Galerkin's technique where testing and basis functions are identical, the elements of the system matrix can be written as

$$
Z_{m n}=\left(\begin{array}{cccc}
A_{x} A_{x} & 0 & 0 & A_{x} V \\
0 & A_{y} A_{y} & 0 & A_{y} V \\
0 & 0 & A_{z} A_{z} & A_{z} V \\
V A_{x} & V A_{y} & V A_{z} & V V
\end{array}\right)
$$

where

$$
\begin{aligned}
\left(A_{i} A_{j}\right)_{m n} & =\hat{\boldsymbol{e}}_{i} \cdot \hat{\boldsymbol{e}}_{j} \int_{V_{m}} N_{m} N_{n} \mathrm{~d} V \\
& -\hat{\boldsymbol{e}}_{i} \cdot \hat{\boldsymbol{e}}_{j} k^{2} \int_{V_{m}} \int_{V_{n}}\left(\epsilon_{n}-1\right) N_{m} N_{n} G \mathrm{~d} V^{\prime} \mathrm{d} V \\
\left(A_{i} V\right)_{m n} & =\hat{\boldsymbol{e}}_{i} \cdot k^{2} \int_{V_{m}} \int_{V_{n}}\left(\epsilon_{n}-1\right) N_{m} \nabla^{\prime} N_{n} G \mathrm{~d} V^{\prime} \mathrm{d} V \\
\left(V A_{j}\right)_{m n} & =-\int_{V_{m}} \int_{V_{n}}\left(\epsilon_{n}-1\right) \cdot N_{m} N_{n} \hat{\boldsymbol{e}}_{j} \cdot \nabla^{\prime} G \mathrm{~d} V^{\prime} \mathrm{d} V \\
(V V)_{m n} & =\int_{V_{m}} N_{m} N_{n} \mathrm{~d} V \\
& +\int_{V_{m}} \int_{V_{n}}\left(\epsilon_{n}-1\right) N_{m} \nabla^{\prime} N_{n} \cdot \nabla^{\prime} G \mathrm{~d} V^{\prime} \mathrm{d} V .
\end{aligned}
$$

The point matching scheme is obtained by replacing the test functions $N_{m}$ with the delta functions $\delta_{m n}\left(\delta_{m n}=1\right.$ when $m=n$ and $\delta_{m n}=0$ when $m \neq n$ ). All singular integrals are evaluated with the singularity extraction method [10]. 


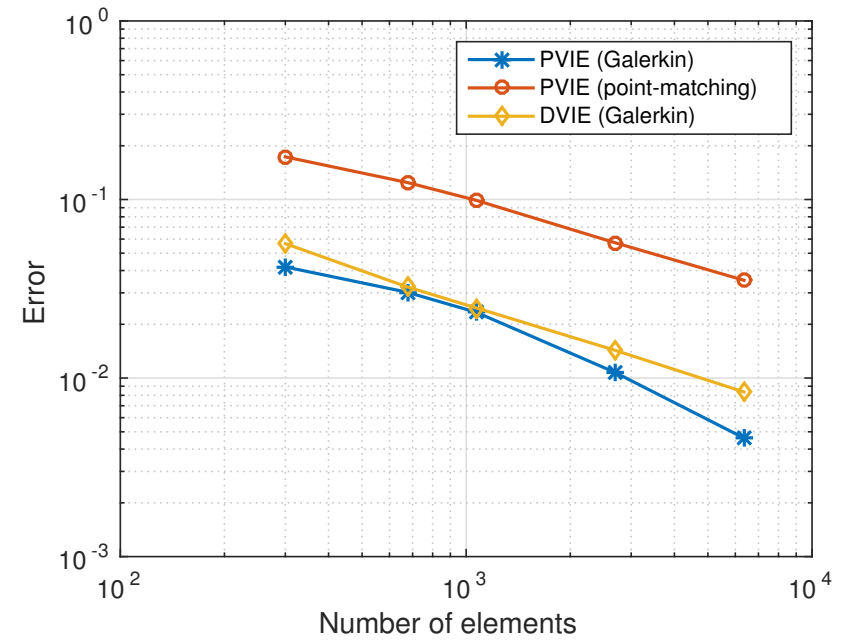

Fig. 1. Convergence of the far-field error for a sphere of size $k a=1$ and $\epsilon_{r}=9+1 i$ computed with three different VIE scheme.

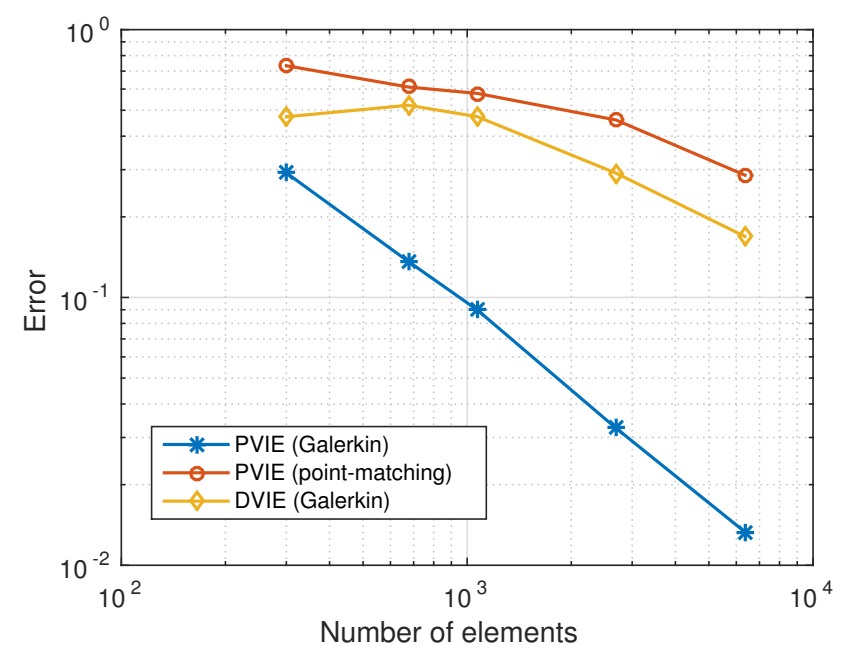

Fig. 2. Same as in Fig. 1 but for a sphere of size $k a=4$ and $\epsilon_{r}=3$.

\section{NUMERICAL EXAMPLES}

In this section, we present numerical examples to validate the proposed method. First, we study the convergence of the solution with respect to the number of unknowns. For the convergence analysis, we apply three different VIE schemes; the potential formulation (PVIE) presented in this paper with the point-matching and Galerkin testing, and the electric-field formulation in which the unknown is the electric flux density (DVIE) discretized by SWG basis and testing functions [1]. The radar cross section (RCS) error quantity is defined as

$$
\text { Error }=\sqrt{\frac{\int_{\gamma}(\sigma-\tilde{\sigma})^{2} \mathrm{~d} S}{\int_{\gamma} \tilde{\sigma}^{2} \mathrm{~d} S}},
$$

where $\sigma$ is the computed RCS, $\tilde{\sigma}$ is the exact RCS of MIE solution, and the integration is over a spherical surface.

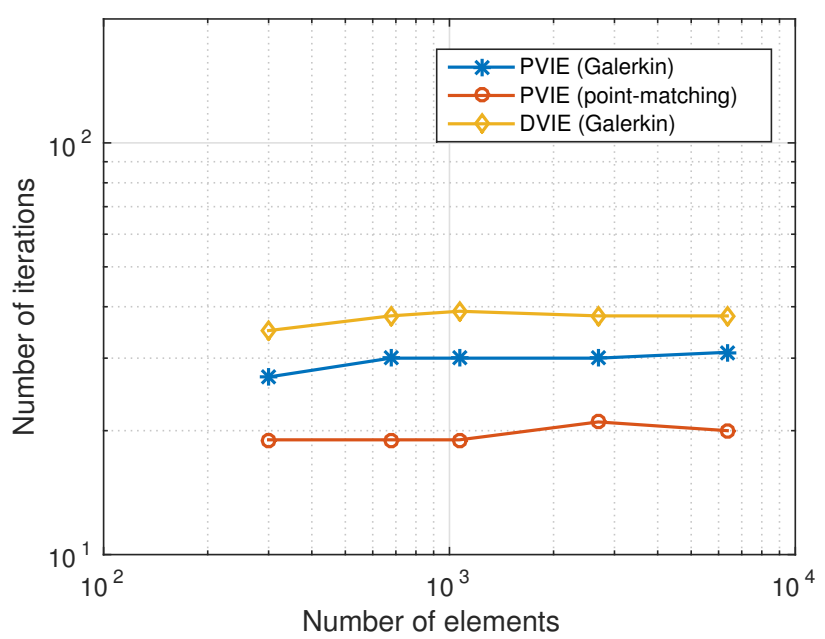

Fig. 3. The number of GMRES(50) iterations required to solve the problem in Fig. 1. The stopping criterion for GMRES is $10^{-5}$.

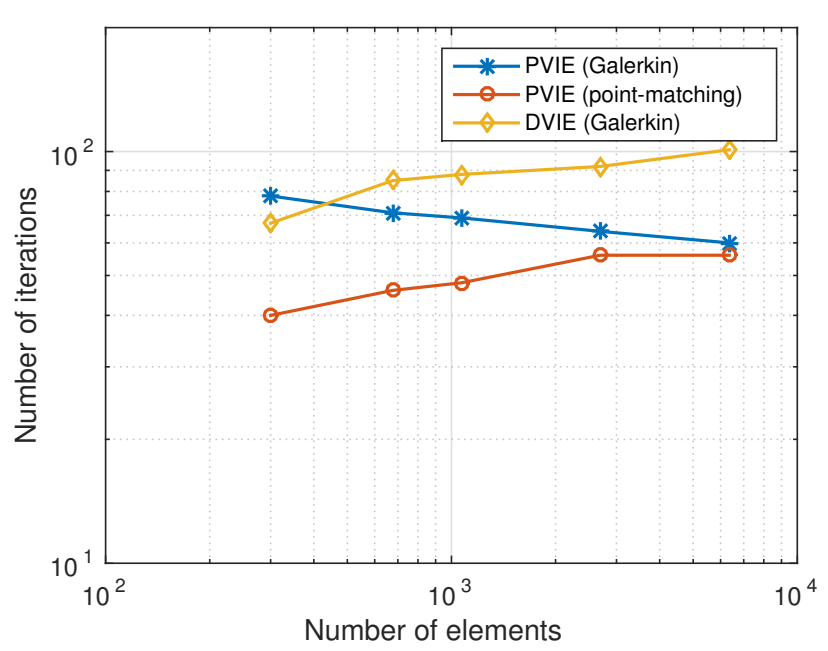

Fig. 4. The number of GMRES(50) iterations required to solve the problem in Fig. 2. The stopping criterion for GMRES is $10^{-5}$.

Figs. 1 and 2 present computed far-field errors. In Fig. 1 the size of the sphere is $k a=1$ and the permittivity $\epsilon_{r}=9+i$. In Fig. 2 the size parameter $k a=4$ and the permittivity $\epsilon_{r}=$ 3 . The systems are solved by the iterative GMRES method and iteration counts are plotted in Figs. 3 and 4. We observe that the PVIE discretized with Galerkin's scheme converges much faster than the point matching discretization. Moreover, the PVIE with Galerkin's testing is more accurate than the standard SWG Galerkin scheme for the DVIE. Interestingly, Galerkin's testing for the PVIE seems to perform well despite the test functions do not span the $L^{2}$-dual space of the range of the integral operator. This may be due to the smooth domain and range since similar observation has been made also for the acoustic surface-integral-equation method when the operator defines mapping between smooth spaces $\left(H^{1 / 2} \rightarrow H^{1 / 2}\right)$ [11]. 


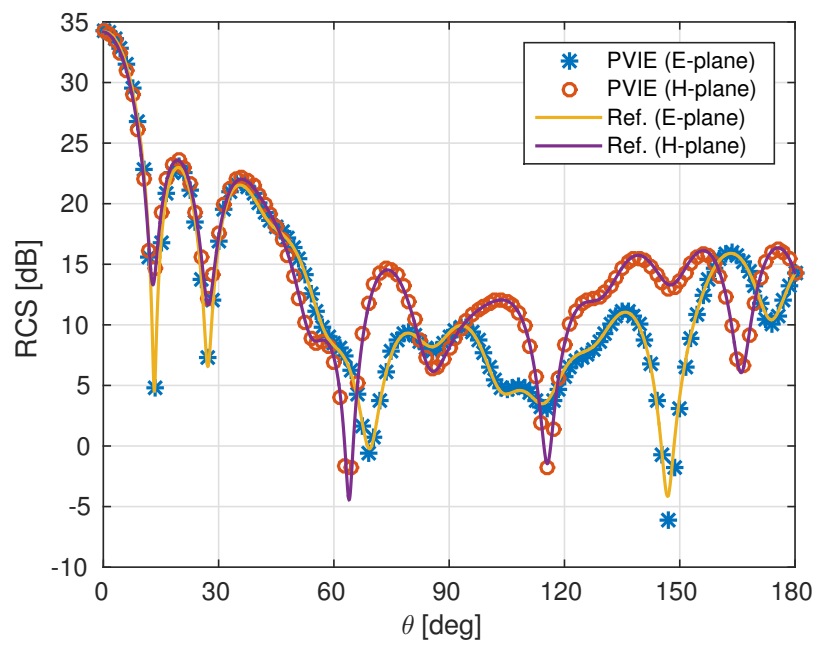

Fig. 5. Computed radar cross section of 512 non-intersecting randomly positioned spheres $\left(r=1\right.$ and $\left.\epsilon_{r}=5+0.1 i\right)$ enclosed by a box of size $20 \times 20 \times 20$ with wavelength $\lambda=2 \pi$. The reference result is calculated using multiple scattering theory of spheres.

Since Galerkin's method shows superior accuracy over the point-matching and the convergence of the iterative solution is rather fast and independent on the mesh density, we accelerate the matrix-vector multiplications required in the iterative solver by the precorrected-FFT method [12]. This allows us to solve much larger problems. As an example, Fig. 5 shows the RCS of 512 non-intersecting randomly distributed equalsized spheres $(r=1)$ in the electric and magnetic field planes. The spheres are packed into a box of size $20 \times 20 \times 20$, and the incident wave is linearly polarized with wavelength $2 \pi$. Each sphere is discretized with 300 tetrahedral elements resulting in 153600 elements and 180224 unknowns in total. The semi-analytical reference result is computed using the multiple scattering theory by spheres [13]. Finally, Fig. 6 displays the electric field components of the internal electric field in a layered sphere computed by the PVIE Galerkin and MIE series. As can be seen The PVIE solution satisfies the interface conditions at $x=-0.5$ and $x=0.5$.

\section{CONCLUSIONS}

The potential volume-integral-equation formulation has been derived and discretized with fully continuous nodal basis functions on tetrahedral mesh. The equations are converted into a discrete system of linear equations employing the pointmatching and Galerkin's method. Galerkin's scheme shows superior accuracy over the point-matching scheme. Moreover, in our test cases, the convergence rate of accuracy of Galerkin's tested PVIE was higher than that of the commonly used fieldbased formulation with SWG-functions and Galerkin's testing.

\section{ACKNOWLEDGMENT}

The research has been funded by the ERC Advanced Grant No 320773 entitled "Scattering and Absorption of ElectroMagnetic waves in ParticuLate media" (SAEMPL).

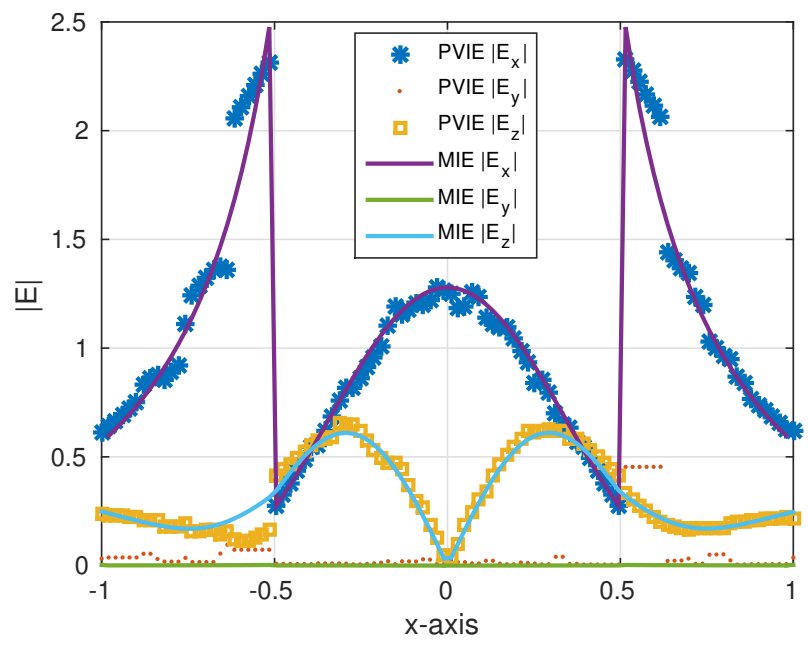

Fig. 6. Internal electric field components along $\mathrm{x}$-axis of a layered sphere with $r_{1}=0.5\left(\epsilon_{r, 1}=50+10 i\right)$ and $r_{2}=1.0\left(\epsilon_{r, 2}=5+1 i\right)$ computed by Galerkin's tested PVIE. The sphere is discretized with 15695 elements.

\section{REFERENCES}

[1] D. Schaubert, D. Wilton, and A. Glisson, "A tetrahedral modeling method for electromagnetic scattering by arbitrarily inhomogeneous dielectric bodies," IEEE Trans. Ant. and Propag., vol. 32, no. 1, pp. 77-85, 1984.

[2] L. Sun and W. Chew, "A novel formulation of the volume integral equation for electromagnetic scattering," Waves in Random and Complex Media, vol. 19, no. 1, pp. 162-180, 2009.

[3] J. Markkanen, C.-C. Lu, X. Cao, and P. Yla-Oijala, "Analysis of volume integral equation formulations for scattering by high-contrast penetrable objects," IEEE Trans. Ant. and Propag., vol. 60, no. 5, pp. 2367-2374, May 2012

[4] M. A. Yurkin, M. Min, and A. G. Hoekstra, "Application of the discrete dipole approximation to very large refractive indices: Filtered coupled dipoles revived," Physical Review E, vol. 82, no. 3, p. 036703, 2010.

[5] P. De Doncker, "A potential integral equations method for electromagnetic scattering by penetrable bodies," IEEE Trans. Ant. and Propag., vol. 49, no. 7, pp. 1037-1042, 2001.

[6] P. DeDoncker, "A volume/surface potential formulation of the method of moments applied to electromagnetic scattering," Engineering analysis with boundary elements, vol. 27, no. 4, pp. 325-331, 2003.

[7] R. Chang and V. Lomakin, "Potential-based volume integral equations," IEEE International Symposium on Antennas and Propagation (APSURSI), pp. 2712-2715, 2011.

[8] M. Adil, "Volume integral equations for the study of electromagnetic scattering by bi-anisotropic objects," Master's thesis, School of Electrical Engineering, Aalto University, Sept. 2013.

[9] J. Markkanen and P. Ylä-Oijala, "Numerical comparison of spectral properties of volume-integral-equation formulations," J. Quant. Spectrosc. Radiat. Transfer, vol. 178, pp. 269-275, Jul. 2016.

[10] S. Järvenpää, M. Taskinen, and P. Ylä-Oijala, "Singularity extraction technique for integral equation methods with higher order basis functions on plane triangles and tetrahedra," International Journal for Numerical Methods in Engineering, vol. 58, no. 8, pp. 1149-1165, 2003.

[11] P. Ylä-Oijala, S. Kiminki, and S. Järvenpää, "Conforming boundary element methods in acoustics," Engineering Analysis with Boundary Elements, vol. 50, pp. 447-458, 2015.

[12] J. R. Phillips and J. K. White, "A precorrected-FFT method for electrostatic analysis of complicated 3-D structures," IEEE Trans. CAD of Integ. Circ. and Sys., vol. 16, no. 10, pp. 1059-1072, 1997.

[13] J. Bruning and Y. Lo, "Multiple scattering of EM waves by spheres part I-multipole expansion and ray-optical solutions," IEEE Trans. Ant. Propag., vol. 19, no. 3, pp. 378-390, May 1971. 